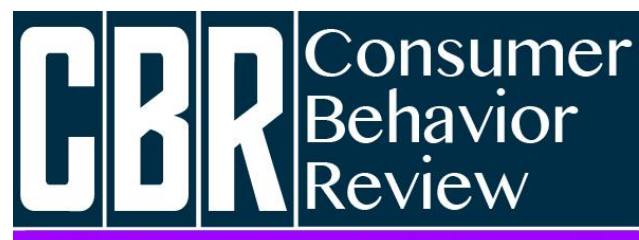

Revista Comportamento do Consumidor
Nunes, M. N., Spanhol-Finocchio, C. P., Quevedo-Silva, F. \& Lima-Filho, D. O. (2018). Relação entre Emoção e Alimentação de Consumidores Idosos. Consumer Behavior Review, 2(2), 5671.
ISSN: 2526-7884

Editor: Prof. Dr. Marconi Freitas da Costa Email da revista: cbr@ufpe.br
Avaliação: Double blind review

Recebido: 24 de janeiro de 2018

Aceito: 01 de junho de 2018

\title{
RELAÇÃO ENTRE EMOÇÃO E ALIMENTAÇÃO DE CONSUMIDORES IDOSOS
}

\author{
Mylena Neres Nunes \\ Caroline Pauletto Spanhol-Finocchio \\ Filipe Quevedo-Silva \\ Dario de Oliveira Lima-Filho
}

\begin{abstract}
Mylena Neres Nunes é Doutoranda em Administração pela Universidade Federal do Mato Grosso do Sul - UFMS. E-mail: mylenaneres@hotmail.com Caroline Pauletto Spanhol-Finocchio é Professora do Programa de PósGraduação em Administração da Universidade Federal do Mato Grosso do Sul - UFMS. E-mail:

carolspanhol@gmail.com.

Filipe Quevedo-Silva é Professor do

Programa de Pós-Graduação em

Administração da Universidade Federal do Mato Grosso do Sul - UFMS. E-mail: quevedo.silva@ufms.br.

Dario de Oliveira Lima-Filho é Professor do Programa de Pós-Graduação em Administração da Universidade Federal do Mato Grosso do Sul - UFMS. E-mail: dariolimafilho@gmail.com.

Os autores agradecem aos avaliadores pelos comentários para melhoria do artigo.
\end{abstract}

\begin{abstract}
Resumo
Considerando o crescimento no número de pessoas com 60 anos de idade ou mais, torna-se relevante conhecer o seu perfil e suas preferências de consumo, uma vez que representam um importante segmento de mercado. Este estudo investigou as emoções evocadas pelos idosos em resposta ao consumo de dois distintos tipos de alimento - churrasco e salada. Também se verificou o impacto das emoções na avaliação, recomendação e intenção de recompra. Foi utilizado o modelo EsSence Profile composto por 39 atributos de emoção classificados em positivos, neutros e negativos. Foram entrevistados 103 idosos. Os resultados revelaram a predominância de emoções positivas, especialmente quando comparadas às negativas, nos dois tipos de alimento. Emoções positivas como satisfeito, feliz, prazeroso e agradável foram experienciadas com maior frequência. Os resultados indicaram que as respostas emocionais foram altamente preditivas para a avaliação, recomendação e intenção de comer novamente os alimentos pesquisados.

Palavras-chave: Comportamento alimentar, Respostas

Emocionais, Alimento, Equação Estrutural, Modelagem.
\end{abstract}

Esta obra está licenciada com uma Licença Creative Commons Atribuição 4.0 Internacional.

\section{INTRODUÇÃO}

A população idosa tem apresentado acelerado crescimento, impulsionado não apenas pela redução da taxa de fertilidade, mas também pelo aumento da expectativa de vida (Organização Mundial da Saúde [OMS], 2015). Uma prova disso é que a maior parte da população mundial tem expectativa de vida superior a 60 anos, fato inédito na história até 
então (United Nations, 2007). A genética, o ambiente físico e social em que o indivíduo esteve inserido influenciam no processo de envelhecimento (OMS, 2015), ou seja, esse processo pode ocorrer de maneiras diferentes entre os indivíduos, variando de uma pessoa para outra. Essa diversidade contribui para a heterogeneidade desse grupo etário (Moschis, 1993), que necessita de produtos mais alinhados às suas necessidades, especialmente aqueles relacionados à alimentação.

Mesmo diante do crescimento e da heterogeneidade da população idosa no mundo, esse grupo vem sendo negligenciado e tem recebido pouca atenção do mercado, principalmente ao se considerar os significados da comida e do ato de comer (Ujil, Jager, Graaf, Waddell \& Kramer, 2014). Destaca-se que a alimentação não é norteada apenas pelas necessidades fisiológicas, mas, também, pelo ambiente, pelo corpo e pelas emoções (Desment \& Schifferstein, 2008). Assim, conhecer as emoções relacionadas à alimentação torna-se relevante e necessário para a elaboração de novos produtos e elaboração de políticas públicas relacionadas à alimentação e nutrição para esse grupo.

Estudos têm se voltado à compreensão das relações entre as emoções e o comportamento alimentar, tendo sido, inicialmente, abordadas as emoções negativas evocadas pelo alimento (Evers, Adriaanse, Ridder \& Witt Huberts, 2013; Laros \& Steenkamp, 2005), e mais recentemente as emoções positivas. Esses estudos identificaram uma considerável predominância de emoções positivas no comportamento alimentar (Desment \& Schifferstein, 2008; King et al., 2010; Macth \& Dettmer, 2006; Evers et al., 2013; Gutjar et al., 2014; Borgogno, 2017), chamada na literatura de assimetria hedônica (Desment \& Schifferstein, 2008).

Outros estudos têm direcionado seus esforços para a criação de modelos que auxiliem na mensuração das emoções evocadas pelos alimentos (King \& Meiselman, 2010, King et al., 2010; Laros \& Steenkamp, 2005; Macht, 2008; Desmet \& Schifferstein, 2008). Nesse contexto, destaca-se o de King e Meilseman (2010) que resultou na criação do modelo EsSence Profile, já utilizado em estudos voltados para a mensuração das emoções na alimentação (Manzocco, Rumignani \& Lagazio, 2013; Cardello et al., 2012; Spinelli et al. 2014; Ujil et al., 2014; Prescott \& Kim, 2017; Kanjanakorn \& Lee, 2017). 0 modelo é composto por 39 termos identificados como mais relacionados à alimentação, já que nem todas as emoções são associadas ao alimento (Laros \& Steenkamp, 2005), sendo classificados em positivos, neutros e negativos.

Diante da breve revisão apresentada, tornase fundamental conhecer e compreender o papel que as emoções desempenham na alimentação. Assim, busca-se responder às seguintes questões de pesquisas: i) Quais emoções são experienciadas pelos idosos durante o consumo de determinados tipos de alimentos? ii) As respostas emocionais são diferentes considerando o tipo de alimento consumido? iii) As emoções influenciam na avaliação e na intenção de comer novamente o alimento? Frente às questões apresentadas, este estudo tem como objetivo mensurar as respostas emocionais dos consumidores idosos em relação a duas categorias de alimentos, churrasco e salada.

A escolha dessas categorias foi orientada por suas distintas características e apelos sensoriais. O churrasco, por exemplo, possui apelo cultural por ser normalmente associado a "comer acompanhado". Além disso, por se tratar de um alimento hedônico, possivelmente tem um forte apelo emocional. Enquanto que as hortaliças, por se tratarem de um alimento importante para a dieta das pessoas, especialmente dos idosos, é um alimento normalmente associado ao consumo funcional. Além disso, considera-se a tendência que as pessoas têm de dar significados sociais, culturais e econômicos distintos para a carne e a salada (Kontukoski, Paakki, Thureson, Uimonen \& Hopia, 2016).

O estudo de King e Meilseman (2010) é utilizado como ponto de partida para esta pesquisa, feita com consumidores idosos. Assim, a tradução, adaptação e validação do EsSence Profile com o público idoso brasileiro constituise a inovação deste estudo e a contribuição para o campo teórico.

Este estudo está dividido em mais cinco seções, além desta introdução. A segunda refere-se ao referencial teórico utilizado na pesquisa, a terceira dedica-se a apresentação da metodologia, a quarta discute os resultados da pesquisa e a quinta aborda as considerações finais. 


\section{REFERENCIAL TEÓRICO}

\section{Alimentação e emoção}

0 alimento afeta a forma como as pessoas se sentem (King et al., 2010) e pode ser não apenas a causa de uma emoção, como também o objeto, ou ainda as duas coisas simultaneamente. Há uma relação de influência bidirecional entre as emoções e a alimentação, isto é, as emoções tanto afetam o comportamento alimentar como também são afetadas por ele (Köster \& Mojet, 2015). Tais fatores têm despertado o interesse dos pesquisadores que nos últimos anos têm voltado seus estudos para o efeito das emoções no comportamento alimentar.

Um entrave para o estudo das emoções é sua ambiguidade. Sabe-se que nem todas as emoções são experimentadas no consumo de alimentos, por exemplo, inveja, orgulho e ciúmes são emoções interpessoais menos associadas à alimentação (Laros \& Steenkamp, 2005). No entanto, ainda não há clareza sobre quão adequadas são as emoções usadas nos estudos existentes para mensurar as respostas ao alimento e sua ingestão (Desment \& Schifferstein, 2008).

Diante da dificuldade em se mensurar as emoções relacionadas à alimentação do ponto de vista do comportamento do consumidor, têm sido crescentes os esforços para a criação de modelos para sua investigação (King \& Meilseman, 2010; Laros, Steenkamp \& 2005; Desmet, 2002; Macht, 2008; Desmet \& Schifferstein, 2008).

Por meio da revisão de literatura foi possível identificar que têm sido conferidas diferentes abordagens para o estudo das influências bidirecionais entre emoções e alimentação. Há estudos que se voltaram para emoções mais específicas, chamadas de nível de base (Laros, 2006), outros usaram um abrangente grupo de emoções (Thomson, Crocker \& Marketo, 2010), há ainda os estudos que abordam emoções específicas como raiva, medo, tristeza e alegria (Macht, 1999), alegria e tristeza (Macht, Roth \& Ellgring, 2002).

É possível ainda dividir os estudos existentes, que abordam simultaneamente emoções e comportamento alimentar, basicamente em dois tipos: os que estudam a interferência da alimentação nas emoções e os que abordam as implicações das emoções na alimentação (Desmet, 2007; Desmet \&
Schifferstein, 2008). Em outras palavras, podese pensar na maneira como o contexto alimentar motiva certa emoção, por exemplo, quando um indivíduo se sente feliz ao comer chocolate e, por outro lado, como a ação das emoções atuam na preferência alimentar, por exemplo, quando uma pessoa com raiva perde a vontade de comer. Existe a possibilidade de diferentes respostas alimentares a diferentes tipos de emoção, essas respostas vão desde a motivação para comer, até a quantidade de alimento ingerida (Macht, 2008), bem como diversas respostas emocionais a distintas propriedades sensoriais (Jiang, King \& Prinyawiwatkul, 2014).

A pesquisa de Desmet e Schifferstein (2008) deu outra abordagem para o estudo das emoções e alimentação. Os autores defendem que para medir as experiências alimentares emocionais não é suficiente identificar apenas as valências, porque uma experiência emocional é mais do que positiva ou negativa, sendo também uma avaliação das circunstâncias em que ocorrem. Assim, os autores identificaram quais emoções eram mais experienciadas em situações de consumo de alimentos e o contexto em que ocorriam. Foi solicitado aos participantes que descrevessem para cada uma das 22 emoções propostas, situações em que as experimentaram. 0 estudo identificou e categorizou cinco fontes de emoções alimentares: propriedades sensoriais (como sabor e aparência), consequências de experiência (saciedade), consequências associadas (imaginar que um alimento engorda, por exemplo), significados sociais (sentir prazer ao comer churrasco por se lembrar dos amigos) e comportamento dos agentes (admiração por quem faz churrasco, por exemplo).

Todos os 42 participantes relataram ao menos uma situação para cada emoção. As emoções que produziram menos exemplos foram ciúmes, raiva e tristeza, já as que geraram mais exemplos foram diversão, satisfação e desejo. Os autores organizaram esses exemplos em cinco categorias: aspecto do produto, agente, tipo de produto, contexto e atividade.

\section{Assimetria hedônica}

A literatura consultada aponta para uma significativa predominância de emoções positivas associadas à alimentação (Desment \& 
Schifferstein, 2008; King \& Meiselman, 2010; Macht \& Dettmer, 2006; Evers et al., 2013), a chamada assimetria hedônica. Desmet (2002) concluiu por meio de sua pesquisa que as emoções positivas são mais ligadas à alimentação do que as negativas, sendo as principais: satisfação, desejo, diversão e prazer, já as desagradáveis foram tédio, decepção e insatisfação.

No estudo de King e Meiselman (2010) também se identificou uma prevalência dos estados emocionais positivos. Entre os 39 atributos emocionais identificados por meio de uma revisão de literatura em dez documentos, apenas três foram classificadas como negativas (bored, disgusted and worried), indicando um número mais expressivo de emoções positivas em resposta aos alimentos.

Contudo, ainda pouco se sabe sobre as emoções e as diferentes maneiras com que cada uma dessas emoções, por exemplo, medo, raiva e desânimo afetam a alimentação (Macht, 2008). A emoção alívio, por exemplo, normalmente aparece quando uma necessidade nutricional (como fome ou sede) é satisfeita (Desment \& Schifferstein, 2008). Sabe-se também que diferentes níveis de emoção têm relação com as mudanças nas atitudes das pessoas com os alimentos (Jiang et al., 2014).

Uma explicação para a tendência de se responder positivamente à ingestão de alimentos, deve-se ao fato de que os indivíduos escolhem alimentos que acreditam que irão provocar emoções agradáveis ao serem degustados, por outro lado, alimentos novos ou desconhecidos provavelmente causariam uma "assimetria hedônica" menos acentuada (Desment \& Schifferstein, 2008).

À medida que um indivíduo se familiariza com um objeto, associações são construídas, sobretudo entre a identidade conferida a ele e outros conceitos existentes na mente. Por exemplo, "comer maçã emagrece", "alimentos ricos em açúcar me relaxam" e "comer sushi é chique" são associações feitas com base em fatores internos e externos (Thomson et al., 2010). Isso quer dizer que se responde não apenas ao produto, mas também as conceptualizações, ou seja, o conceito conferido a eles tem grande responsabilidade quanto ao tipo de emoção que será conferida ao alimento (Jiang et al., 2014).
As associações feitas com o alimento desempenham um importante papel nos estados emocionais e não há como separá-las, isto é, as emoções que são sentidas ao ingerir um alimento podem conter fatores diretamente ligados à comida como o sabor, ou indiretamente como, por exemplo, o medo de engordar (Desment, 2002; Desment \& Schifferstein, 2008). Assim, as respostas emocionais aos alimentos podem receber interferência não apenas das propriedades sensoriais do alimento, mas também de características individuais que explicam as diferentes respostas alimentares afetivas despertadas por alimentos com propriedades sensoriais distintas (Rozin, 2015).

\section{Alimentação, emoção e idosos}

Os idosos sentem muita dificuldade ao ter que lidar com as modificações de seus hábitos, sobretudo alimentares. A temporalidade alimentar na última etapa da vida, assim como nas demais, afeta os fatores que compõem a alimentação, por exemplo, a quantidade de tomadas alimentares já não é a mesma, o volume das refeições, alimentos antes permitidos tornam-se proibidos e novos alimentos são inseridos.

Destaca-se que os idosos são considerados os consumidores do futuro e formam um grupo heterogêneo (Ujil, Jager, de Graaf, Meilseman \& Kramer, 2016), são consumidores experientes (Ujil et al., 2017), com necessidades alimentares mais complexas que as anteriores e consideravelmente distintas.

Estudos anteriores já se voltaram para a compreensão do comportamento alimentar de idosos (Lima et. al., 2008), considerando fatores relacionados ao contexto (por exemplo, onde e com quem), ao alimento e ao consumidor (por exemplo, aspectos demográficos). Os autores identificaram que os idosos consideram a qualidade nutricional dos alimentos a variável mais importante e que mais de $70 \%$ deles fazem de três a quatro refeições por dia (Lima et al., 2008).

Apesar da importância desse grupo de consumidores, poucos são os estudos voltados para a compreensão da alimentação do ponto de vista das emoções. Apenas a gerontografia e a psicografia não são suficientes para fornecer percepções específicas das necessidades e 
desejos alimentares dos idosos (Ujil et al., 2014), além do mais, a idade é um aspecto importante para a mediação de emoções alimentares (Ujil et al., 2017).

Considerando o exposto, e com o objetivo de verificar a influência das emoções na avaliação, intenção de comer novamente (recompra) e recomendação, foi elaborada a seção 2.4.

\section{Consequentes das emoções}

A Avaliação dos alimentos consumidos foi mensurada englobando aspectos de atitude e lealdade atitudinal. As Atitudes podem ser consideradas predisposições estáveis de comportamentos subseqüentes, o que a torna capaz de prever com uma certa eficácia os comportamentos de consumo nos estudos de marketing (Mitchell \& Olson, 1981). A Lealdade é considerada um "fenômeno multidimensional complexo" (Jacoby \& Kyner, 1973, p. 1), englobando não apenas comportamentos de recompra, mas também aspectos cognitivos, emoções e comportamentos (Dick \& Basu, 1994). Diante disso, apresenta-se a primeira hipótese de pesquisa:

H1: As emoções influenciam positivamente a avaliação do cliente.

Tanto as atitudes como a lealdade atitudinal tem uma importante relação com a intenção de recompra, já que a motivação de comprar novamente um produto é uma das possíveis respostas de consumo fornecida pelos consumidores às condições necessárias e suficientes para definir e conceituar lealdade. Ou seja, lealdade implica em duração, sendo uma condição a recompra (Jacoby \& Kyner, 1973). Face ao exposto, apresenta-se a segunda hipótese de pesquisa:

H2: As emoções influenciam positivamente na intenção de comer novamente.

A recomendação ou o "boca a boca" é reconhecido como uma importante e eficaz ferramenta de recomendação, em que o consumidor naturalmente emite opiniões negativas ou positivas a respeito do produto, atuando como uma ferramenta de marketing e meio de se medir a satisfação (Kozinets, Valck, Wojinick \& Wilner, 2010). Nesse sentido, apresenta-se a terceira hipótese da pesquisa.

H3: As emoções influenciam positivamente a recomendação.

\section{MÉTODO DA PESQUISA \\ Participantes}

A amostra utilizada na pesquisa foi não probabilística. Para tanto, foram selecionados por conveniência 106 indivíduos idosos, com idade igual ou superior a 60 anos, moradores da cidade de Campo Grande - MS. O nível de confiança da amostra é de $95 \%$ e do erro amostral de 10\%. As entrevistas ocorreram em locais com com grande movimentação de pessoas, como ruas e centros de convivência. Foram considerados válidos 103 questionários, sendo homens $(n=53)$ e mulheres $(n=50)$. A maioria dos participantes eram consumidores regulares (mais de uma vez por mês) das duas categorias de alimentos, isto é, churrasco e salada. A Tabela 1 apresenta as características dos participantes.

\section{Instrumento de pesquisa}

Foi utilizado um questionário estruturado como instrumento de coleta de dados em dois formatos, que se diferiam apenas pela ordem em que os alimentos foram apresentados (em um o churrasco era o primeiro alimento e no outro a salada), isso foi feito com a finalidade de minimizar os efeitos que um possível cansaço ou tédio pudesse causar na qualidade das respostas.

O questionário foi dividido em três partes. $\mathrm{Na}$ primeira etapa foi identificada a frequência de consumo e a aceitabilidade dos alimentos (churrasco e salada). Também foram mensuradas as variáveis consequentes das emoções, por meio de uma escala Likert de cinco pontos, apresentadas no Quadro 1: avaliação, intenção de comer novamente e intenção de recomendar. A escala de avaliação foi elaborada com base em Quevedo-Silva et al. (2013) e Lopes et al. (2009), combinando fatores de atitude e lealdade atitudinal. 
Tabela 1

Características sócio demográficas dos participantes $(\mathrm{n}=103)$

\begin{tabular}{ccc}
\hline Fator & Nível & \% da amostra \\
\hline \multirow{2}{*}{ Sexo } & Masculino & 51,5 \\
& Feminino & 48,5 \\
\hline \multirow{3}{*}{ Idade } & $60-65$ anos & $38,80 \%$ \\
& $66-70$ anos & $35,90 \%$ \\
& $71-76$ anos & $17,50 \%$ \\
& $\geq 77$ anos & $7,80 \%$ \\
\hline \multirow{3}{*}{ Estado civil } & Solteiro & 9,7 \\
& Casado & 45,6 \\
& Viúvo & 17,5 \\
Come churrasco mais de uma vez por mês & 68,9 \\
\hline \multirow{2}{*}{ Come salada mais de uma vez por mês } & 7,8 \\
\hline
\end{tabular}

Fonte: Elaborado pelos autores.

A escala de Intenção de comer novamente foi adaptada das escalas desenvolvidas por Mittal, Kumar e Tsiros (1999) e Lemon, White \& Winer (2002). A escala de recomendação foi adaptada da escala de Maxham \& Netemeyer (2002).

A aceitabilidade do produto foi identificada na primeira parte, atendendo a recomendação dos autores de identificar a aceitabilidade antes da mensuração das emoções (King \& Meilseman, 2010). Sabe-se que há uma forte correlação entre as emoções e a aceitabilidade do alimento, o que torna imprescindível a complementaridade entre teste de emoção e testes de aceitabilidade (King \& Meilseman, 2010).

Quadro 1 - Indicadores de avaliação, intenção de consumo/compra e de recomendação

\begin{tabular}{|c|l|}
\hline Código & \multicolumn{1}{|c|}{ Indicadores da A valiação } \\
\hline Aval1 & Quando eu penso em reunião com amigos/família, churrasco é minha primeira opção \\
\hline Aval2 & Eu gosto de churrasco \\
\hline Aval3 & Eu acredito que churrasco é o meu prato preferido \\
\hline Código & \multicolumn{1}{c|}{ Indicadores de consumo e compra } \\
\hline Intenção1 & Eu pretendo comer churrasco no futuro \\
\hline Intenção2 & Eu pretendo comer churrasco com mais frequência \\
\hline Intenção3 & Eu pretendo aumentar o valor das minhas compras relacionadas ao churrasco \\
\hline Código & \\
\hline recom1 & Eu pretendicadores de intenção de recomendação bem sobre churrasco com as pessoas \\
\hline recom2 & Eu pretendo recomendar churrasco para meus amigos e parentes \\
\hline recom3 & Se alguém pedir meu conselho, eu pretendo recomendar churrasco \\
\hline
\end{tabular}

Fonte: elaborado pelos autores. 
A segunda parte do questionário teve como finalidade identificar as emoções evocadas pelos alimentos. Para tanto, foi utilizado o modelo EsSence Profile (King \& Meilseman, 2010). O modelo EsSence Profile é composto por 39 termos de emoção que foram traduzidos para o português por um especialista. Após a tradução um pré-teste com 10 idosos foi realizada para averiguar a adequabilidade do instrumento de coleta de dados. Os termos e suas classificações são apresentados no Quadro 4.

Quadro 4 - Grupo total de variáveis do construto emoção e sua classificação

\begin{tabular}{|c|c|c|}
\hline Construto & Afeto & Indicadores \\
\hline \multirow{38}{*}{ Emoções } & \multirow{24}{*}{ Positivo } & Agradável (pleasant) \\
\hline & & Alegre (joyful) \\
\hline & & Amigável (friendly) \\
\hline & & Amoroso (loving) \\
\hline & & Animado (active) \\
\hline & & Ativo (energetic) \\
\hline & & Aventureiro (adventurous) \\
\hline & & Bondoso (good-natured) \\
\hline & & Calmo (calm) \\
\hline & & Carinhoso (affectionate) \\
\hline & & Completo (whole) \\
\hline & & Contente (glad) \\
\hline & & $\begin{array}{l}\text { Divertido (merry) } \\
\text { Entusiasmado (enthusiastic) }\end{array}$ \\
\hline & & $\begin{array}{l}\text { Entusiasmado (enthusiastic) } \\
\text { Feliz (happy) }\end{array}$ \\
\hline & & Frágil (tender) \\
\hline & & Generoso (good) \\
\hline & & Livre (free) \\
\hline & & Nostálgico (nostalgic) \\
\hline & & Prazeroso (pleased) \\
\hline & & Quente (warm) \\
\hline & & Satisfeito (satisfied) \\
\hline & & Seguro (secure) \\
\hline & & Sereno (tame) \\
\hline & & Tranquilo (peaceful) \\
\hline & \multirow{3}{*}{ Negativo } & Enojado (disgusted) \\
\hline & & Entediado (bored) \\
\hline & & Preocupado (worried) \\
\hline & \multirow{11}{*}{ Neutro } & Agressivo (aggressive) \\
\hline & & Ansioso (eager) \\
\hline & & Compreensivo (understanding) \\
\hline & & Culpado (guilty) \\
\hline & & Educado (polite) \\
\hline & & Estável (steady) \\
\hline & & Interessado (interested) \\
\hline & & Leve (mild) \\
\hline & & Ousado (daring) \\
\hline & & Selvagem (wild) \\
\hline & & Silencioso (quiet) \\
\hline
\end{tabular}

Fonte: King e Meiselman (2010).

As emoções são classificadas no modelo em positivas, negativas e neutras. No entanto, dois termos foram reclassificados neste estudo com base no modelo de Sosa, Cardinal, Contarini \& Hough (2015) e Cardello et al. (2012) (Quadro 5 ), foram eles agressivo e culpado. Além desses, 
um terceiro atributo reclassificado foi "frágil", de acordo com a comprovação estatística que forneceu a segurança necessária para a mudança. Em síntese, os termos "culpado", "agressivo" e "frágil" foram reclassificados como negativos.

A última parte do questionário levantou os dados demográficos, como renda, idade, estado civil, escolaridade e profissão.

Quadro 5 - Justificativa para a reclassificação dos termos "culpado", "frágil" e "agressivo"

\begin{tabular}{|c|c|c|}
\hline Termo & Alteração no modelo & Justificativa \\
\hline Agressivo & De neutro para negativo & $\begin{array}{c}\text { Questões semânticas foram o principal fator na decisão de alteração da } \\
\text { classificação do termo, bem como outros estudos que também } \\
\text { classificaram o termo como negativo (Sosa et al., 2015; Cardello et al, } \\
\text { 2012). Os testes estatísticos também apontaram para uma maior ligação } \\
\text { do termo com a valência negativa. }\end{array}$ \\
\hline Culpado & De neutro para negativo & $\begin{array}{c}\text { O significado conferido ao termo na língua portuguesa foi a motivação } \\
\text { inicial para a reclassificação. Outros estudos também conferiam valência } \\
\text { 2012; Edwards, Hartwel \& Brown, 2013). Os testes estatísticos } \\
\text { identificaram uma maior ligação do termo com a valência negativa. }\end{array}$ \\
\hline Frágil & $\begin{array}{c}\text { De positivo para } \\
\text { negativo }\end{array}$ & $\begin{array}{c}\text { O termo tem significado negativo em português, daí a necessidade de } \\
\text { alteração da reclassificação, que mais tarde foi corroborada pela análise } \\
\text { estatística. }\end{array}$ \\
\hline
\end{tabular}

Fonte: Elaborado pelos autores.

\section{Procedimentos}

Sabe-se que o tipo de estímulo interfere nas respostas emocionais do alimento, ou seja, se forem utilizados teste de sabor, imagens, odores ou o nome do produto as respostas irão variar (King, Meilseman \& Carr, 2010).

As respostas emocionais são mais intensas quando o estímulo é feito apenas pelo nome do alimento, isso ocorre porque o respondente tende a idealizar o produto, trazer memórias antigas ao invés de ser realista (King, Meilseman \& Carr, 2010; Cardello et al., 2012), bem como produz respostas mais estáveis ao longo do tempo quando comparado ao estímulo de provar o alimento, por exemplo (Jiang et al., 2014). Diante disso, optou-se por realizar o estímulo por meio do nome do produto.

0 nome do alimento (churrasco e salada) foi mencionado várias vezes ao longo da aplicação do questionário, com a finalidade de que o participante mantivesse o alimento em mente durante toda a aplicação do questionário, já que o momento ideal para questionar os entrevistados é quando o estímulo ocorre ou logo após (King, Meilseman \& Carr, 2010).
As respostas foram dadas por meio de uma escala Likert de cinco pontos, que partia de 1 "discordo totalmente" para 5 "concordo totalmente". Os dados foram analisados com o software SPSS. Buscando caracterizar a amostra foi realizada uma análise descritiva dos dados em que foi analisado desvio padrão e a média realizando comparações pareadas.

A análise dos dados foi feita por meio da modelagem de equações estruturais que objetivou validar as relações entre as variáveis latentes do modelo, verificando como cada termo de emoção refletia na avaliação, intenção de recompra e recomendação.

\section{ANÁLISE E DISCUSSÃO Análise descritiva}

A aceitabilidade foi mensurada por meio de uma escala Likert de cinco pontos que variou de "discordo totalmente" (1) a "concordo totalmente" (5). Os participantes em sua maioria afirmaram gostar de ambos os produtos, ou seja, responderam concordar totalmente (5) ou parcialmente (4) que gostavam de churrasco e salada (Tabela 2). 
Com relação à frequência de consumo dos alimentos, o consumo de salada foi superior ao do churrasco. Aproximadamente 98,1\% dos entrevistados afirmaram comer salada mais de uma vez por mês, enquanto $68,9 \%$ disseram comer churrasco mais de uma vez por mês.

Tabela 2

Aceitabilidade do churrasco e salada

\begin{tabular}{ccc} 
& Média & Desvio \\
\hline Churrasco & 3,932 & 1,2854 \\
Salada & 4,087 & 1,1808 \\
\hline
\end{tabular}

Fonte: Elaborada pelos autores

Os entrevistados usaram os 39 termos de emoção para expressar sua intensidade em relação aos dois grupos alimentares propostos. A Figura 1 mostra as emoções experienciadas pelos respondentes. É notável a predominância de emoções positivas nas respostas emocionais ao churrasco. Todos os dez termos com maiores médias são positivos. Foram eles: satisfeito, feliz, prazeroso, contente, agradável, animado, ativo, divertido, amigável e tranquilo, respectivamente. Este viés de positividade já foi encontrado em diversos estudos com outros grupos etários (Borgogno, Cardello, Favotto \& Piasentier, 2017; Cardello et al, 2012; Edwards, Hartwell \& Brown, 2013; Gutjar et al., 2015), inclusive com indivíduos da terceira idade (Ujil et al., 2014).

Em resposta à salada, emoções positivas e neutras estão entre as predominantes. A emoção neutra "leve" foi a que obteve maior média, seguida pela positiva "tranquilo", as seguintes foram: feliz, estável, seguro, calmo, agradável, educado, sereno, alegre e silencioso, respectivamente. Ou seja, apesar de menos acentuada do que a identificada no churrasco, também foi encontrada a assimetria hedônica nas respostas emocionais dos participantes para a salada, com a presença também de termos neutros entre as maiores médias.

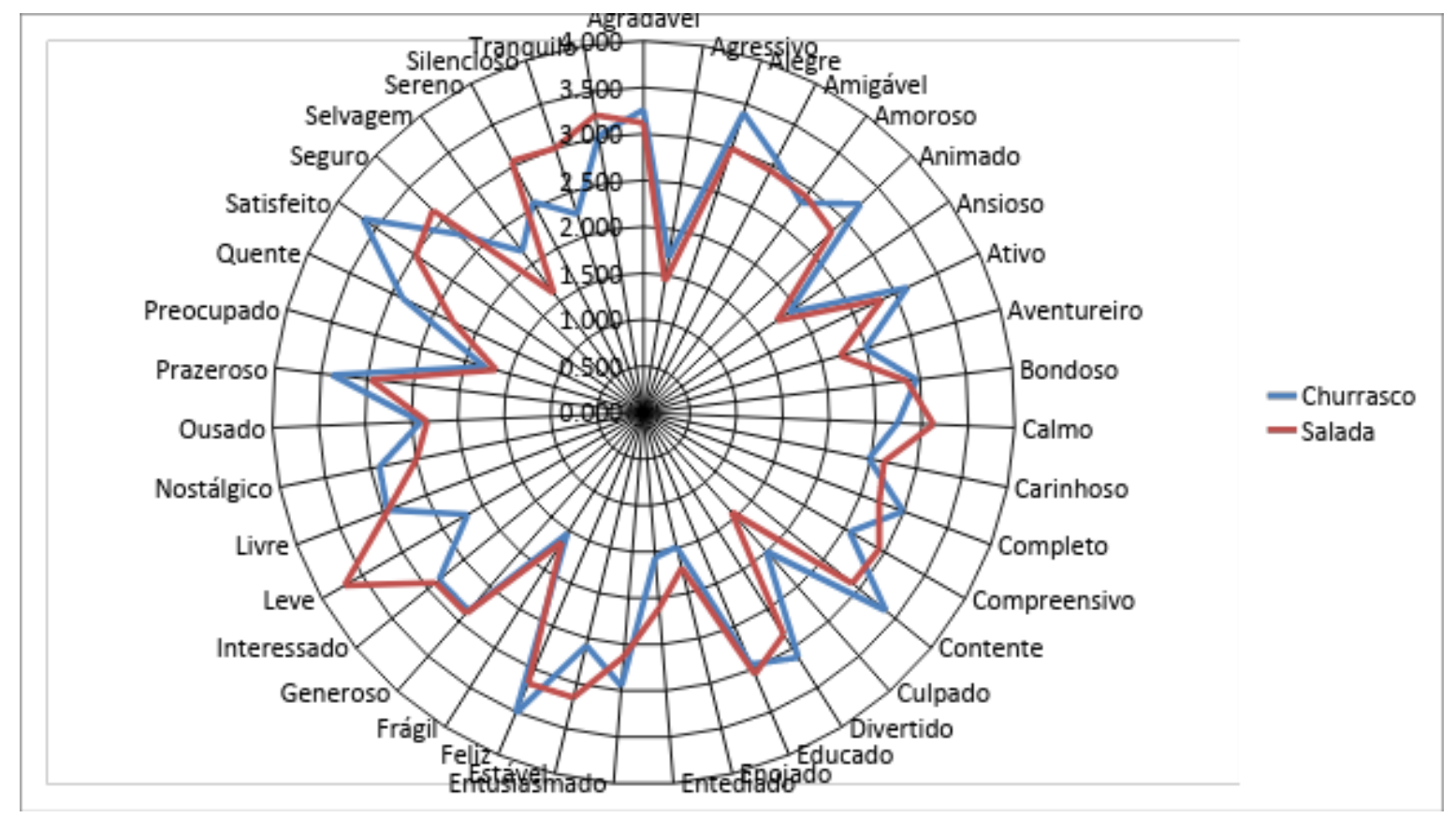

Figura 1 - Médias das respostas dos entrevistados para cada uma das emoções Fonte: Elaborado pelos autores.

\section{Análise multivariada:}

\section{modelagem de equações estruturais}

A relação entre os construtos de avaliação, intenção de comer novamente e recomendação foi realizada por meio da técnica de Modelagem de Equações Estruturais (SEM) no programa SmartPLS 3. A técnica de Modelagem de Equações Estruturais tem sido amplamente utilizada no campo das ciências sociais aplicadas (Hair, Black, Babin, Anderson \& 
Tatham, 2009) e é capaz de explicar relações entre múltiplas variáveis, além de examinar simultaneamente uma série de relações de dependência inter-relacionadas de diversas variáveis latentes (Hair et al., 2009).

\section{Estruturação do modelo}

A primeira etapa da análise foi a estruturação do modelo no software SmartPLS 3 em que os construtos foram relacionados aos termos de emoção, ou seja, aos indicadores. Após rodar o SEM foi possível iniciar as análises de ajuste do modelo, começando pela avaliação dos modelos de mensuração, que foram validados por meio da validade convergente, discriminante e significância dos caminhos.

\section{Validade convergente}

Ao se avaliar a Average Variance Extracted (AVE), após a primeira tentativa de SEM para o alimento churrasco, observou-se um número abaixo do aceitável, isto é, AVE $<0,50$. Diante disso, foi preciso retirar termos de emoção que não se relacionavam com seus construtos, ou seja, foram excluídos os termos com as menores cargas fatoriais, o que culminou com a retirada das variáveis "preocupado", "agressivo" e "frágil", respectivamente.

\section{Tabela 3}

Valores da qualidade de ajuste do modelo MEE: churrasco

\begin{tabular}{cccc}
\hline Convergente & Alpha & $\begin{array}{c}\text { Composite } \\
\text { Reliability }\end{array}$ & AVE \\
\hline Intenção & 0.861 & 0.915 & 0.782 \\
Avaliação & 0.864 & 0.917 & 0.787 \\
Negativas & 0.572 & 0.778 & 0.539 \\
Neutras & 0.819 & 0.872 & 0.577 \\
Positivas & 0.939 & 0.950 & 0.703 \\
Recomendação & 0.925 & 0.952 & 0.869 \\
\hline
\end{tabular}

Fonte: Elaborado pelos autores.

Esses três termos também foram identificados na análise descritiva entre os cinco com menores médias, ou seja, os idosos participantes da pesquisa alegaram nunca experimentar ou experimentar pouco essas emoções com relação ao churrasco. Após a retirada desses termos com menor correlação, os índices de ajuste convergente foram considerados satisfatórios. Os valores da qualidade de ajuste referente ao churrasco podem ser conferidos na Tabela 3.

Tabela 4

Resultado da análise convergente: salada

\begin{tabular}{cccc}
\hline Convergente & Alpha & $\begin{array}{c}\text { Composite } \\
\text { Reliability }\end{array}$ & A VE \\
\hline Intenção & 0.884 & 0.928 & 0.812 \\
Avaliação & 0.879 & 0.925 & 0.805 \\
Negativas & 0.557 & 0.767 & 0.526 \\
Neutras & 0.769 & 0.850 & 0.589 \\
Positivas & 0.810 & 0.886 & 0.723 \\
Recomendação & 0.900 & 0.937 & 0.832 \\
\hline
\end{tabular}

Fonte: elaborado pelos autores.

Após a primeira tentativa de SEM com os dados do alimento salada, também foram observados números com $\mathrm{AVE}<0,50$. Os termos com menores cargas fatoriais retirados foram: selvagem, ansioso, silencioso e ousado. Ansioso e selvagem tiveram médias baixas na análise descritiva. A análise convergente do estudo referente à salada pode ser conferida na Tabela 4.

\section{Verificação da validade discriminante}

A terceira etapa da avaliação dos modelos buscou identificar as distinções existentes entre os construtos similares (Hair et al., 2009), identificando as variáveis mais responsáveis pelas diferenças encontradas (Malhotra, 2012).

Por meio da verificação das cargas cruzadas dos dados dos dois grupos de alimentos foi possível identificar que algumas variáveis latentes (VLs) possuíam carga superior em outros construtos que não os seus originais. Concluída a verificação discriminante do churrasco e da salada foi possível constatar que os construtos "neutra" e "positiva" apresentaram baixa independência, o que aponta para uma não diferenciação da classificação de alguns atributos de emoção entre neutros e positivos. Assim, os termos que apresentavam maior ligação com o grupo do qual não faziam parte foram excluídos.

Os termos excluídos do churrasco foram: tranquilo, calmo, sereno, bondoso, seguro, generoso, carinhoso, divertido, amoroso, amigável, quente, agradável, silencioso, aventureiro, completo, livre e nostálgico. Após a exclusão destes termos, as cargas fatoriais de 
todas as VLs ficaram superiores em seus construtos originais (Tabela 5).

Tabela 5

Resultado da análise de validade discriminante: churrasco

\begin{tabular}{ccccccc}
\hline Discriminante & Intenção & A valiação & Negativas & Neutras & Positivas & Recomendação \\
\hline Intenção & 0.885 & & & & & \\
Avaliação & 0.747 & 0.887 & & & & \\
Negativas & -0.581 & -0.567 & 0.734 & & & \\
Neutras & 0.583 & 0.638 & -0.373 & 0.760 & & \\
Positivas & 0.696 & 0.738 & -0.505 & 0.757 & 0.838 & \\
Recomendação & 0.735 & 0.841 & -0.555 & 0.609 & 0.754 & 0.932 \\
\hline
\end{tabular}

Fonte: elaborado pelos autores.

Já da salada foram: nostálgico, aventureiro, sereno, ativo, amoroso, calmo, tranquilo, aventureiro, bondoso, agradável, amigável, generosos, carinhoso, seguro, completo, quente, livre, prazeroso, entusiasmado, divertido, estável e alegre (Tabela 6).

Tabela 6

Resultado da análise de validade discriminante: salada

\begin{tabular}{ccccccc}
\hline Discriminante & Intenção & A valiação & Negativas & Neutras & Positivas & Recomendação \\
\hline Intenção & 0.901 & & & & & \\
Avaliação & 0.719 & 0.897 & & & & \\
Negativas & -0.593 & -0.601 & 0.725 & & & \\
Neutras & 0.446 & 0.598 & -0.503 & 0.767 & & \\
Positivas & 0.583 & 0.704 & -0.591 & 0.755 & 0.850 & \\
Recomendação & 0.642 & 0.810 & -0.481 & 0.567 & 0.676 & 0.912 \\
\hline
\end{tabular}

Fonte: elaborado pelos autores.

\section{Significância dos caminhos}

Após a conclusão dos modelos de mensuração foi feita a análise dos caminhos do modelo proposto, objetivando verificar se havia relação ou não entre os construtos. A análise estrutural do churrasco pode ser vista na Tabela
7. Os três construtos relacionados às emoções neutras não apresentaram coeficientes estimados significativos a um nível de $5 \%$. Isso aponta que as emoções neutras não influenciam na recomendação, avaliação e intenção de comer novamente do churrasco.

Tabela 7

Significância dos caminhos: churrasco

\begin{tabular}{cccccc}
\hline & Original Sample & Sample Mean & Standard & T Statistics & P Values \\
\hline Negativa - Intenção & -0.310 & -0.313 & 0.078 & 3.994 & 0.000 \\
Negativa - Avaliação & -0.263 & -0.263 & 0.085 & 3.094 & 0.002 \\
Negativa - Recomendação & -0.235 & -0.234 & 0.084 & 2.806 & 0.005 \\
Neutras - Intenção & 0.137 & 0.132 & 0.141 & 0.977 & 0.329 \\
Neutras - Avaliação & 0.192 & 0.187 & 0.125 & 1.534 & 0.126 \\
Neutras - Recomendação & 0.096 & 0.096 & 0.113 & 0.848 & 0.397 \\
Positivas - Intenção & 0.436 & 0.441 & 0.145 & 3.007 & 0.003 \\
Positivas - Avaliação & 0.460 & 0.469 & 0.123 & 3.750 & 0.000 \\
Positivas -Recomendação & 0.563 & 0.567 & 0.116 & 4.839 & 0.000 \\
\hline
\end{tabular}

Fonte: Elaborado pelos autores. 
Semelhante ao que ocorreu com o churrasco, as emoções neutras também obtiveram coeficientes não significativos a um nível de 5\% para a salada, ou seja, as emoções neutras não influenciam na avaliação, recomendação e na intenção (Tabela 8).

Tabela 8

Significância dos caminhos: salada

\begin{tabular}{cccccc}
\hline Mean & Original Sample & Sample Mean & Standard & T Statistics & P values \\
\hline Negativa - Intenção & -0.385 & -0.380 & 0.124 & 3.107 & 0.002 \\
Negativa - Avaliação & -0.214 & -0.225 & 0.066 & 3.262 & 0.001 \\
Negativa - Recomendação & -0.115 & -0.115 & 0.106 & 1.082 & 0.280 \\
Neutras - Intenção & -0.037 & -0.037 & 0.115 & 0.320 & 0.749 \\
Neutras - Avaliação & -0.014 & -0.014 & 0.109 & 0.127 & 0.899 \\
Neutras - Recomendação & 0.116 & 0.116 & 0.122 & 0.958 & 0.339 \\
Positivas - Intenção & 0.383 & 0.383 & 0.138 & 2.767 & 0.006 \\
Positivas - Avaliação & 0.668 & 0.668 & 0.111 & 6.022 & 0.000 \\
Positivas-Recomendação & 0.520 & 0.520 & 0.135 & 3.852 & 0.000 \\
\hline
\end{tabular}

Fonte: Elaborado pelos autores.

Com relação às emoções positivas, todos os coeficientes apresentaram valores significativos a um nível de confiança de $5 \%$ para os dois grupos de alimentos, e com base nestes resultados, percebe-se que as emoções positivas estão positivamente relacionadas com a intenção de comer novamente, recomendar e com a avaliação, ou seja, as respostas emocionais que elas evocam têm interferência nas variáveis, sendo inclusive os resultados mais expressivos, fato este que corrobora com os resultados encontrados na análise descritiva. A relação "positivas-recomendação" apresentou os resultados mais expressivos para o churrasco, o que aponta para a intenção de recomendar que as emoções positivas evocam. Já para a salada as emoções positivas tiveram ligação mais intensa com a Avaliação.

\section{MODELOS}

Os modelos produziram bom ajuste, indicando que as respostas emocionais foram altamente preditivas para os indicadores de avaliação, recomendação e intenção de comer novamente.

\section{Modelo - Churrasco}

Como indicado na Figura 2, "alegre", "animado", "ativo", "contente", "entusiasmado", "feliz", "prazeroso" e "satisfeito", estavam positiva e significativamente relacionados com a avaliação, intenção de comer novamente e de recomendar.

Os resultados indicaram também uma relação mais significativa entre emoções positivas e os três construtos, o que demonstra uma maior interferência de emoções positivas na recomendação, intenção de comer novamente e avaliação. A relação entre as emoções positivas e o construto recomendação, foi a que apresentou maiores resultados quando comparada a todos os demais, ou seja, os idosos entrevistados demonstraram que as emoções positivas interferem significativamente na recomendação do churrasco. 0 construto com maior relação com as emoções negativas foi a intenção de comer novamente, o que significa que quanto mais presentes as emoções negativas culpa, enojado e entediado, maior é a intenção de recomendar negativamente o churrasco. 


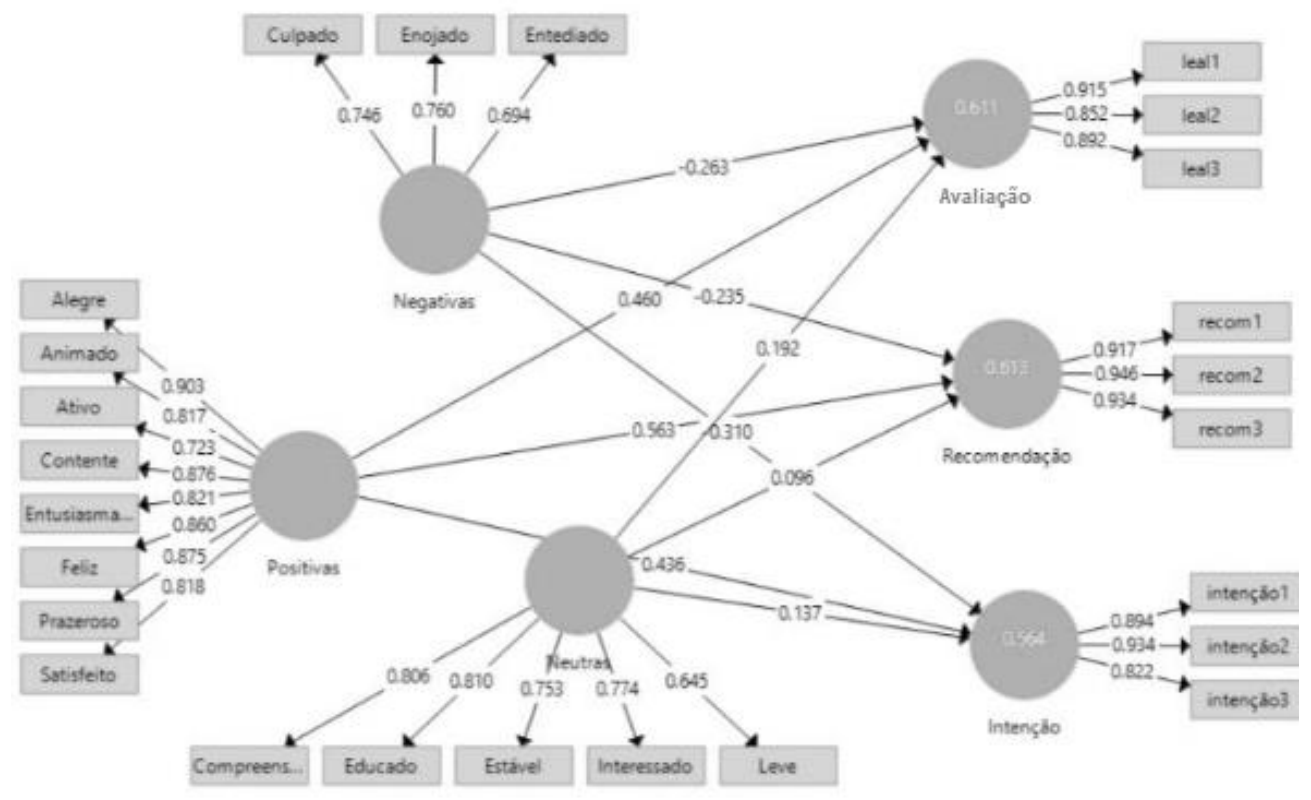

Figura 2 - Modelo estrutural churrasco Fonte: Elaborado pelos autores.

\section{Modelo - Salada}

A emoção "leve" foi a emoção com maior avaliação para a salada na análise descritiva e também apresentou boa avaliação na análise multivariada, assim como "educado". Apesar disso, foi possível identificar por meio do teste de significância dos caminhos, que emoções neutras não têm relação significativa com a avaliação, recomendação e intenção de comprar novamente, ou seja, as emoções mais associadas com a salada não têm interferência significativa na escolha de ingerir esse alimento, por exemplo.

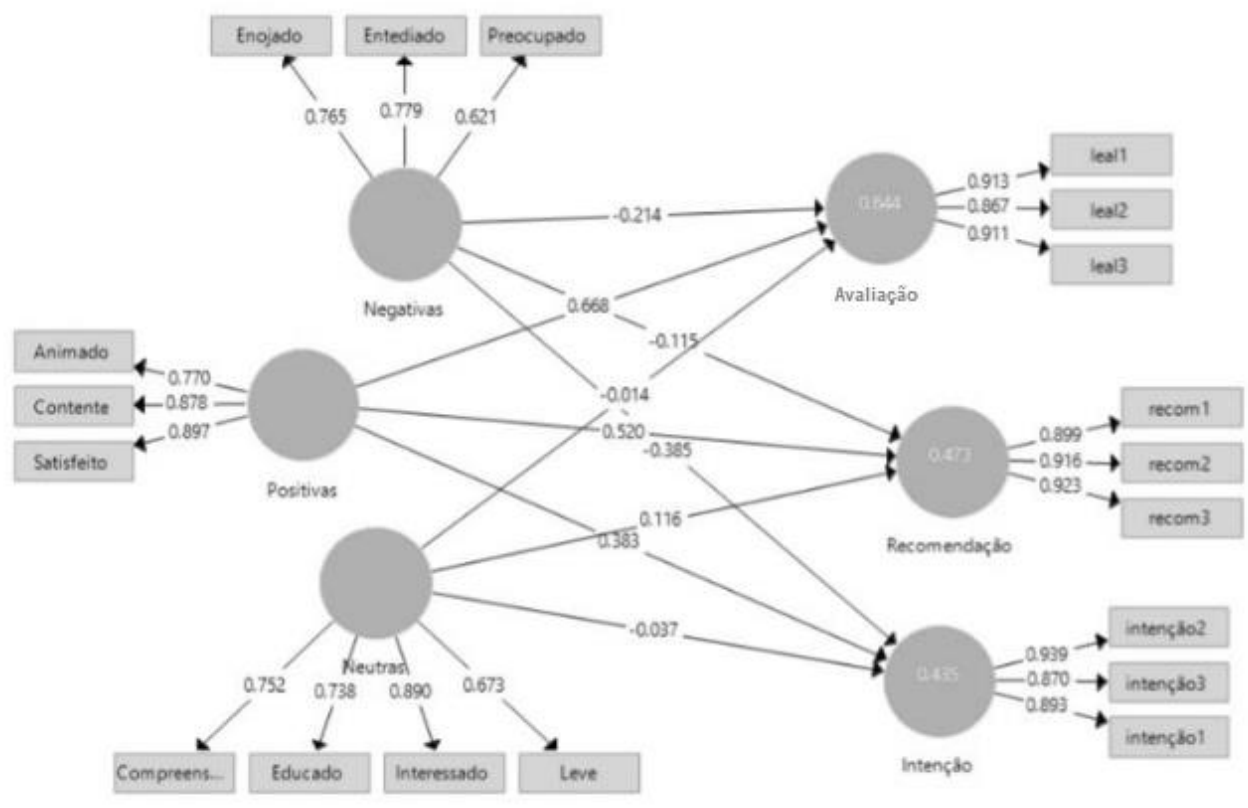

Figura 3 - Modelo estrutural: salada Fonte: Elaborado pelos autores 
As emoções positivas "animado", "contente" e "satisfeito" que na análise descritiva apresentaram médias abaixo de três, foram os termos com maior influência na avaliação e principalmente recomendação, isso aponta para uma baixa avaliação dos termos que influenciam o comportamento. Curiosamente, apesar de os participantes terem relatado gostar de salada e pretender indicar salada e comer novamente, por exemplo, as emoções positivas (satisfeito, contente e animado) tiveram resultados próximos aos das emoções negativas (enojado, entediado e preocupado) na influência de comer novamente. Enquanto que as emoções positivas demonstraram influenciar positivamente na intenção de comer novamente, as negativas influenciaram negativamente.

As emoções positivas afetam mais na recomendação, sendo essa a interferência mais forte encontrada no modelo, ou seja, as emoções positivas interferem significativamente na recomendação da salada. Além disso, ao analisar os betas nota-se que têm pesos diferentes para os atributos negativos e positivos, sendo que os positivos exercem maior interferência nos três atributos.

\section{CONSIDERAÇÕES FINAIS}

Este estudo buscou investigar as emoções evocadas por alimentos e o seu efeito sobre o comportamento de consumidores idosos, usando como estímulo dois grupos de alimento: salada e churrasco.

Este estudo mostrou que as emoções desempenham um importante papel na escolha e ingestão de alimentos por adultos mais velhos, capazes de, por meio do estímulo correto, responder com variados termos de emoção para descrever aspectos emocionais evocados (Ferrarini et al., 2010). Observou-se que os idosos associam diferentes emoções aos alimentos, provavelmente relacionando-as não apenas com as propriedades sensoriais, mas também, com seus significados e agentes (Desmet \& Schifferstein, 2008) e que conferem diversos significados à alimentação devido às variadas memórias e experiências que tiveram com ela ao longo dos anos (Ujil et al., 2014).

Os resultados revelaram um viés de positividade nas avaliações, ou seja, houve predominância de emoções positivas. Confirma- se também que diferentes alimentos despertam diferentes emoções, isso está de acordo com o encontrado em pesquisas anteriores (King \& Meiselman, 2010).

Assim, a análise sensorial / emocional pode ser interessante para caracterização das preferências de idosos para os mais variados tipos de alimentos e não apenas churrasco e salada. Esse tipo de análise pode ser utilizada como uma importante ferramenta na diferenciação de alimentos voltados para consumidores idosos.

Diante do protagonismo das emoções provocadas pelo alimento em consumidores idosos, mostra-se importante alinhar as características emocionais do alimento com as sensoriais, de maneira que os alimentos sejam concebidos e comercializados com base também no impacto emocional, o que minimizaria erros nas estratégias de marketing, especialmente ao se desenvolverem produtos alimentares para os idosos que visem suprir necessidades nutricionais específicas.

Alguns aspectos devem ser observados em estudos futuros. Abordar alimentos distintos não apenas nas propriedades sensoriais, como também na aceitabilidade, uma vez que pode gerar resultados diferentes, especialmente com relação à assimetria hedônica. Um esforço na tentativa de comparar os resultados entre homens e mulheres também ofereceriam uma contribuição interessante.

\section{REFERÊNCIAS}

Borgogno, M., Cardello, A.V., Favotto, S. \& Piasentier, E. (2017, May). An emotional approach to beef evaluation. Meat Science, 127(1), 1-5.

Cardello, A.V., Meilseman, H.L., Schutz, H.G., Craig. C., Given, Z., Lesher, L.L. \& Eicher, S. (2012, June). Measuring emotional responses to foods and food names using questionnaires. Food Quality and Preference, 24(2), 243-250.

Den Ujil, L.C., Jager, G., de Graaf, C., Waddell, J., \& Kremer, S. (2014, September). It is not just a meal, it is an emotional experience - A segmentation of older persons based on the emotions that they associate with mealtimes. Appetite, 83(1), 287-296.

Den Ujil, L.C., Jager, G., de Graaf, C., Meilseman, H.L. \& Kremer, S. (2016, March). Emotion, olfaction, and age: A comparison of self-reported foodevoked emotion profiles of younger adults, 
older normosmic adults, and older hyposmic adults. Food Quality and Preference, 48(1), 199-209.

Den Ujil, L.C., Jager, G., de Graaf, C. \& Kremer, S. (2017, Março). Applying mealtime functionality to tailor protein-enriched meals to older consumer segments. Appetite, 107(1), 613-622.

Desment, P. M. A. \& Schifferstein, H. N. J. (2008, March - May). Sources of positive and negative emotions in food experience. Appetite, 50(1), 2-3.

Dick, A.S. \& BASU, K. (1994, March). Customer loyalty: Toward an integrated conceptual framework. Journal of the Academy of Marketing Science, 22(2), 99-113.

Edwards, J. S.A., Hartwell, H. J. \& Brown, L. (2013, October). The relationship between emotions, food consumption and meal acceptability when eating out of the home. Food Quality and Preference, 30(1), 22-32.

Evers, C., Adriaanse, M., de Ridder, D. T. \& de Witt Huberts, J.C. (2013, Abril). Good mood food. Positive emotion as a neglected trigger for food intake. Appetite, 68(1), 1-7.

Ferrarini, R., Carbognin, C., Casarotti, E.M., Nicolis, E., Nencini, A. \& Meneghini, A.M., (2010, October). The emotional response to wine consumption. Food Quality and Preference, 21(1), 720-725.

Gutjar, S., de Graaf, C., Kooijman, V., de Wijk, R. A., Nys, A., ter Horst, G. J. \& Jager, G. (2015, October). The role of emotions in food choice and liking. Food Research International, 76(2), 216-223.

Hair, J.F. Jr., Black, W.C., Babin, B.J., Anderson, R.E. \& Tatham, R.L. (2009). Análise multivariada de dados. Porto Alegre: Bookman.

Jacoby, J. \& Kyner, D. B. (1973, February). Brand Loyalty vs. Repeat Purchasing Behavior. Journal of Marketing Research, 10(1), 1-9.

Jiang, Y., King, J. M. \& Prinyawiwatkul, W. (2014, March). A review of measurement and relationships between food, eating behavior and emotion. Food Science \& Technology, 36(1), 15-28.

Kanjanakorn, A. \& Lee, J. (2017, March). Examining emotions and comparing the essense profile $\AA$ and the coffee drinking experience in coffee drinkers in the natural environment. Food Quality and Preference, 56(Part A), 69-79.

King, S C. \& Meiselman, H. L. (2010, March). Development of a method to measure consumer emotions associated with foods, Food Quality and Preference 21(2), 168-177.

King, S. C., Meiselman, H. L. \& Carr, B. T. (2010, December). Measuring emotions associated with foods in consumer testing. Food Quality and Preference, 21(8), 1114-1116.

King, S. C., Meiselman, H. L. \& Carr, B. T. (2013, April). Measuring emotions associated with foods: Important elements of questionnaire and test design. Food Quality and Preference, 28(1), 8 16.

Kontukoski, M., Paakki, M., Thureson, J., Uimonen, H., \& Hopia, A. (2016, Julho). Imagined salad and steak restaurants: Consumers' colour, music and emotion associations with different dishes. International Journal of Gastronomy and Food Science, 4(1), 1-1.

Köster, E. P. \& Mojet, J. (2015, October). From mood to food and from food to mood: A psychological perspective on the measurement of food-related emotions in consumer research. Food Research International, 76(2), 180-191.

Kozinets, R. V., Valck, K., Wojnicki, A. C. \& Wilner, S. J. S. (2010, March). Networked Narratives: Understanding Word-of-Mouth Marketing in Online Communities. American Markting Association, 74(1), 71-89.

Laros, F. J. M. \& Steenkamp, J E.M. (2005, October). Emotions in consumer behavior: a hierarchical approach. Journal of Business Research, 58(10), 1437-1445.

Lemon, K. N., White, T. B., Winer, R. S. (2002, January). Dynamic customer relationship management: incorporating future considerations into the service retention decision. Journal of Marketing, 66(1), 1-14.

Lima, D. O. Filho, Sproesser, R.L., Lima, M.F.E.M. \& Luchesse, T. (2008, Outubro - Dezembro). Comportamento alimentar do consumidor idoso. Revista de Negócios, 13(4), 27-39.

Macht, M. (1999, AUGUST). Characteristics of eating in anger, fear, sadness and joy. Appetite, 33(1), 129-139.

Macht, M \& Dettmer, D. (2006, May). Everyday mood and emotions after eating a chocolate bar or an apple. Appetite, 46(3), 332-336.

Macht, M. (2008, January). How emotions affect eating: A five-way model. Appetite, 50(1), 111.

Macht, M., Roth, S. \& Ellgring, H. (2002, October). Chocolate eating in healthy men during experimentally induced sadness and joy. Appetite, 39(2), 147-158.

Malhotra, N. K. (2012). Pesquisa de Marketing. Uma orientação aplicada. Porto Alegre: Bookman.

Manzocco, L., Rumignani, A. \& Lagazio, C. (2013, April). Emotional response to fruit salads with different visual quality. Food Quality and Preference, 28(1), 17-22.

Maxham, J. G. \& Netemeyer, R. G. (2002, October). A longitudinal study of complaining customers 
evaluations of multiple service failures and recovery efforts. Journal of Marketing, 66(4), 57-71.

Mittal, V., Kumar, P. \& Tsiros, M. (1999, April). Attribute-level performance, satisfaction, and behavioral intentions over time: a consumption-system approach. Journal of Marketing, 63, 88-101.

Mitchell, A. A. \& Olson, J. C. (1981). Are product attribute beliefs the only mediator of advertising effects on brand attitudes? Journal of Marketing Research, 18, 318-332.

Moschis, G.P. (1993, December). Gerontographics: a scientific approach to analysing and targeting the mature market. Journal of Consumer Marketing, 10(3), 43-53.

Organização Mundial Da Saúde. (2015). Relatório Mundial de envelhecimento e saúde. Disponível em: http://apps.who.int/iris/bitstream/10665/1 86463/1/9789240694811_eng.pdf. Acesso em 20 jan. 2018.

Prescott, J, \& Kim, 0. K. (2017, July). Emotional responses to sweet foods according to sweet liker status. Food Science \&Technology, 59, 1-7. Quevedo-Silva, F., Freire, O. B. L. \& LOPES, E, L. (2013, October). Antecedentes da lealdade no e-commerce: o papel chave da satisfação dos consumidores. In: XVI SEMEAD - Seminário em Administração, São Paulo.

Rozin,P (2015, March). Food preferences, psychology and physiology. International Encyclopedia of the Social \& Behavioral Science, 9(2), 296-299.

Sosa, M., Cardinal, P., Contarini., A., Hough. G. (2015, October). Food choice and emotions: Comparison between low and middle income populations. Food Research International, 76(2), 253-260.

Spinelli, S., Masi, C., Zoboli, G.P., Prescott, J. \& Monteleone, E. (2014, June). Emotional responses to branded and unbranded foods. Food Quality and Preference, 42(1), 1-11.

Thomson, D. M. H., Crocker, C. \& Marketo, C. G. (2010, December). Linking sensory characteristics to emotions: An example using dark chocolate. Food Quality and Preference, 21(1), 11171125.

United Nations. (2007). World economic and social survey 2007: development in an ageing world. Disponível em: http://www.un.org/en/development/desa/p olicy/wess/wess_archive/2007wess.pdf. Acesso em 20 jan. 2018.

\title{
Relationship between emotion and feeding of elderly consumers
}

\begin{abstract}
Considering the growth in the number of people aged 60 and over is relevant to know the profile and their consumption preferences, since they represent an important market segment. This study investigated the emotions in response to the consumption of two distinct types of food - barbecue and salad by the elderly. In addition, we verified the impact of the emotions on the indicators of loyalty, recommendation and repurchase intention. The EsSence Profile model was used. It is composed of 39 attributes of emotion classified as positive, neutral and negative. In total 103 elderly people were interviewed. The results revealed the predominance of positive emotions, especially when compared to negative ones. Positive emotions such as satisfied, happy, pleasurable, and enjoyable were experienced more often. The results indicated that the emotional responses were highly predictive for the indicators of loyalty, recommendation and intention to eat again the types of food researched.
\end{abstract} Keywords: Food Behavior, Emotional Responses, Food, Structural Equation, Modeling. 